\title{
Parâmetros morfométricos e biológicos de populações monoclonais e multiclonais do pulgão-gigante-do-pinus
}

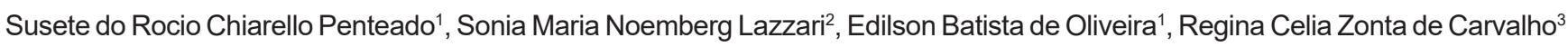 \\ ${ }^{1}$ Embrapa Florestas, Estrada da Ribeira, Km 111, C P 319, CEP 83411-000, Colombo, PR, Brasil \\ ${ }^{2}$ Universidade Federal do Paraná, Av. Cel. Francisco H. dos Santos, 100, Jardim das Americas, CEP 81531-980, Curitiba, PR, Brasil \\ ${ }^{3}$ Centro de Diagnóstico Marcos Enrietti, R. Jaime Balão, 575, Hugo Lange, CEP 80040-340, Curitiba, PR, Brasil
}

*Autor correspondente:
susete.penteado@embrapa.br

Termos para indexação:

Fecundidade

Progênie

Cinara atlantica

Index terms:

Fecundity

Progenie

Cinara atlantica

Histórico do artigo:

Recebido em 17/11/2015

Aprovado em 02/12/2016

Publicado em 30/12/2016

doi: $10.4336 / 2016 . p f b .36 .88 .1110$
Resumo - O pulgão-gigante-do-pinus, Cinara atlantica, originário da América do Norte, foi introduzido no Brasil na década de 1990 e encontra-se amplamente distribuído nos plantios comerciais de pínus. Este estudo teve como objetivo correlacionar os parâmetros morfométricos de insetos oriundos de colônias mono e multiclonais de $C$. atlantica com seu desempenho reprodutivo em mudas de viveiros comerciais e em progênies de Pinus taeda. Foi registrado o número de ninfas produzidas fêmea dia $^{-1}$. Após a morte destas fêmeas, foram realizadas medições dos caracteres morfológicos, utilizando o programa, Axion Vision 4,5. O comprimento da tíbia, fêmur posterior e antena foram os parâmetros morfométricos que apresentaram maior correlação com o comprimento do corpo dos insetos, representando $98 \%$ da variação total. Com os valores destas variáveis foi criada uma nova variável, denominada "tamanho do inseto", que foi utilizada para comparação dos tratamentos. Os insetos de colônias multiclonais apresentaram os maiores valores para a maioria dos parâmetros morfométricos e maior produção de descendentes. Insetos maiores e maior produção de ninfas foram observados em mudas originárias dos viveiros comerciais, sugerindo que as progênies estudadas podem apresentar algum fator de resistência que as torna candidatas para uso em programas de manejo integrado desta praga.

\section{Morphometric and biological parameters of monoclonal and multiclonal populations of giant conifer aphid}

\begin{abstract}
The giant conifer aphid, Cinara atlantica, originated from North America, was introduced in Brazil in the 1990's and became widely distributed on commercial areas of Pinus. This study was conducted in order to correlate morphometric parameters of mono and multiclonal $C$. atlantica lineage with its reproductive performance on plant progenies and commercial seedlings. The number of nymphs produced by female by day were registered. After death, measurements of morphological characters were carried out using the program Axion Vision 4.5. Antenna, hind femur and tibia lengths showed the highest correlation with aphids body length, representing $98 \%$ of total variation. Based on these variables a new variable was defined as 'insect size', which was used to compare treatments. Multiclonal insects had the highest values for most morphometric parameters and largest progeny production. The biggest insects and highest nymph production were observed on commercial seedlings of both nurseries, suggesting that these pine progenies may show some resistance against $C$. atlantica attack what make them good candidates for integrated pest management programs.
\end{abstract}




\section{Introdução}

Cinara atlantica Wilson, 1919 (Hemiptera: Aphididae) é nativa da América do Norte e foi introduzida no Brasil na década de 1990, atacando somente plantas do gênero Pinus. Mudas e plantios novos são mais sensíveis à infestação, o que pode comprometer o desenvolvimento da planta, causar entortamento do fuste e superbrotação, sendo que ataques intensos em plantios estressados podem ocasionar a morte de plantas (Penteado et al., 2000).

Fatores como alta fecundidade e reprodução por partenogênese garantem o sucesso destes insetos como praga (Carver et al., 1991). Porém, a sua capacidade reprodutiva é afetada por diferentes fatores, como a espécie e cultivar da planta hospedeira, temperatura (Dixon, 1987; Kocourek et al., 1994), se são originários de criações monoclonais (ninfas provenientes de uma única fêmea), ou multiclonais (ninfas provenientes de diferentes fêmeas), dentre outros. Mesmo quando se alimentam de árvores da mesma espécie de conífera, o ritmo de crescimento e a fecundidade dos afídeos podem ser significativamente alteradas entre árvores diferentes (Carter \& Watson, 1991).

Plantas com maior concentração de nutrientes, como o nitrogênio, também podem favorecer o desenvolvimento dos insetos, como observado por Hogendorp et al. (2006) e Camargo (2007).

A não-preferência de um afídeo por determinados hospedeiros pode resultar na redução da fecundidade total e diária, como também na redução do seu tamanho e aumento da mortalidade (Kennedy \& Kishaba, 1977). O tamanho dos afídeos é um fator importante, por apresentar significativa influência nas táticas reprodutivas do inseto (Dixon, 1998). Os afídeos maiores atingem a maturidade e iniciam a reprodução antes, resultando em um período reprodutivo mais prolongado e aumento no seu desempenho biológico (Traicevski \& Ward, 1994).

Para muitos insetos herbívoros, as variáveis alométricas, tais como o comprimento da tíbia posterior, estão fortemente correlacionadas com a sua fecundidade (Carter et al., 1991). Uma relação é dita alométrica quando uma característica física ou fisiológica sofre variação com o tamanho do organismo, apresentando assim grande importância nos estudos de comparação e da história de vida das espécies (Begon et al., 1986).

Para fornecer subsídios ao Programa de Manejo Integrado dos pulgões-gigantes-do-pínus, este trabalho teve como objetivos correlacionar os parâmetros morfométricos de colônias multi e monoclonais de C. atlantica com o seu desempenho reprodutivo em mudas de viveiro comerciais e em diferentes progênies de Pinus taeda.

\section{Material e métodos}

Os estudos foram conduzidos no Laboratório de Entomologia Florestal da Embrapa Florestas, localizado em Colombo, PR.

As mudas de Pinus taeda de viveiros comerciais, com 6 meses de idade, foram obtidas em empresas do Rio Grande do Sul e Paraná. Mudas de cinco progênies de P. taeda, também com seis meses de idade, faziam parte de um programa de seleção genética de uma empresa florestal localizada no Estado de Santa Catarina.

Os afídeos foram coletados aleatoriamente em plantios de pínus, levados ao laboratório e transferidos para mudas de $P$. taeda. As mudas infestadas foram mantidas em sala climatizada $\left(20^{\circ} \mathrm{C} \pm 2{ }^{\circ} \mathrm{C}\right.$, umidade relativa de $70 \% \pm 10 \%$ e fotofase de $14 \mathrm{~h}$ ) e acondicionadas em gaiolas cilíndricas de PVC $(37 \mathrm{~cm}$ x $10 \mathrm{~cm})$, com a parte superior de plástico transparente e lateralmente com três aberturas recobertas com tela de malhas bem fechadas anti-afídeo. As mudas foram suspensas por um disco de espuma para impedir o escape do inseto para o tubete. Na porção inferior de cada tubete foi acoplado um frasco contendo água, para manter a umidade do solo. Sempre que necessário, realizava-se a reposição das mudas, para garantir a manutenção das colônias de Cinara atlantica.

Para a obtenção de insetos de colônias multiclonais de $C$. atlantica, foram selecionadas, ao acaso, diferentes fêmeas que vieram do campo, e destas selecionadas ninfas, as quais foram individualizadas nas gaiolas, conforme descrito anteriormente. As ninfas foram acompanhadas até o estágio adulto para o registro diário dos diferentes parâmetros avaliados. O delineamento experimental foi inteiramente ao acaso. $\mathrm{O}$ número de ninfas (repetições) utilizadas em cada tratamento foi: T1 - 20; T2 - 25; T3 - 24; T4 - 18; T5 - 23; T6 - 22 e $\mathrm{T} 7-24$.

Para a obtenção de insetos de colônias monoclonais de $C$. atlantica, foram selecionadas 10 fêmeas da criação descrita anteriormente, as quais foram individualizadas nas gaiolas. Destas, foi selecionada a fêmea que apresentou melhor produção de ninfas; estas ninfas foram individualizadas para os testes monoclonais. 
Em uma primeira etapa, as fêmeas oriundas de colônias multiclonais foram testadas nos seguintes tratamentos: T1 e T2 - mudas provenientes de viveiro do RS e PR, respectivamente; T3, T4, T5, T6 e T7 - representando as cinco progênies de $P$. taeda. $\mathrm{O}$ delineamento experimental também foi inteiramente ao acaso. O número de ninfas (repetições) utilizadas em cada tratamento foi o mesmo relatado anteriormente.

Os parâmetros avaliados foram a produção média de ninfas dia $^{-1}$ para cada fêmea e a produção total de ninfas fêmea ${ }^{-1}$. Após a morte da fêmea adulta, esta foi conservada em álcool $70 \%$ para as medições dos caracteres morfométricos.

Em uma segunda etapa, foram realizados seis tratamentos utilizando fêmeas da colônia monoclonal: T1 - mudas de $P$. taeda de viveiro do RS e T3, T4, T5, T6, T7 - progênies de $P$. taeda de SC. O tratamento, T2 foi eliminado deste ensaio, pois na ocasião não havia disponibilidade de mudas. A metodologia e os parâmetros avaliados foram os mesmos utilizados na primeira etapa. O delineamento experimental também foi inteiramente ao acaso. O número de ninfas (repetições) utilizadas em cada tratamento foi: T1 - 19; T3 - 21; T4 - 19; T5 - 32; T6 - 23 e T7 - 23.

A avaliação dos parâmetros morfométricos foi conduzida no Laboratório de Fitoparasitologia do Centro de Diagnóstico Marcos Enrietti (CDME-SEAB). Os insetos fixados em álcool $70 \%$ foram montados em lâminas semipermanentes e, para isso, os espécimes foram colocados sobre uma lâmina com uma gota de ácido lático para a clarificação do inseto. Sobre a lâmina foi colocada uma lamínula, sendo que, entre elas, colocou-se um pequeno pedaço de lamínula, que serviu de suporte para não danificar as estruturas do inseto. Imagens de cada inseto foram capturadas com o sistema Window Canon Power Shot A620, sob microscópio estereoscópico, e as imagens armazenadas com o programa Axion Vision 4,5. Foram realizadas as medições dos seguintes caracteres morfológicos: largura da cabeça, comprimentos do corpo, antena, tíbia posterior, fêmur posterior, tarsômero II da perna posterior e segmentos rostrais IV $+\mathrm{V}$ (Figura 1).

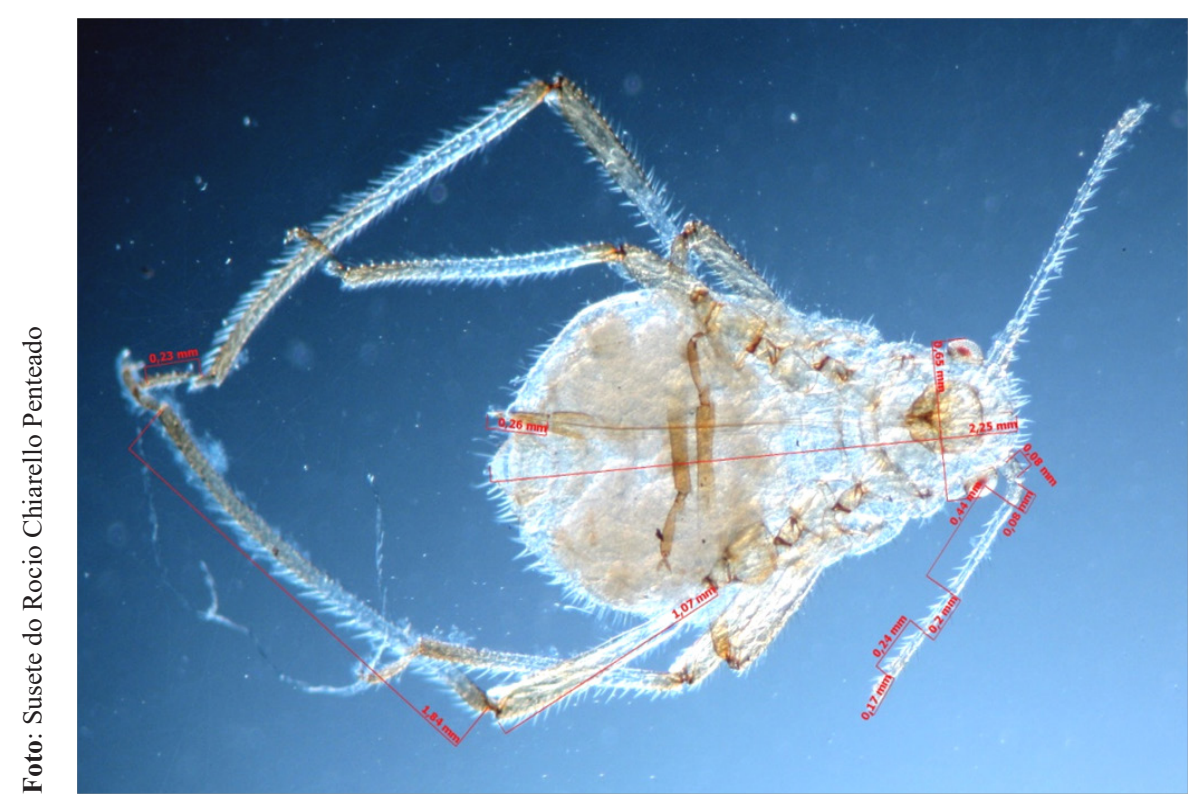

Figura 1. Parâmetros morfométricos utilizados para as medições de adultos ápteros de Cinara atlantica criados em mudas de Pinus taeda, Colombo, PR.

O número de insetos mensurados variou nos diferentes tratamentos porque alguns espécimes não apresentavam todas as estruturas selecionadas para as medições, sendo, neste caso, descartados.

- criação multiclonal: T1 - 11; T2 - 10; T3 - 6; T4 - 4; T5 - 11; T6 - 7 e T7 - 8;
- criação monoclonal: T1 - 16; T3 - 4; T4 - 3; T5 -7 ; T6 - 5 e T7 - 5 .

Os dados foram analisados utilizando-se o teste de Duncan ao nível de $5 \%$ de probabilidade, de coeficientes de correlação, teste t e por análise de componentes principais. 


\section{Resultados e discussão}

A maior produção de ninfas fêmea-1 ocorreu, para os insetos multiclonais, nas mudas de viveiros comerciais (T1 e T2), sendo, entretanto, estatisticamente diferentes apenas do T3 (Tabela 1). Para os insetos monoclonais, a maior produção de ninfas fêmea ${ }^{-1}$ ocorreu nas mudas de viveiro do RS (T1), que diferiu estatisticamente dos demais tratamentos (Tabela 2). Zaleski (2003), utilizando insetos de colônias multiclonais mantidos a $20^{\circ} \mathrm{C}$, observou, para Cinara atlantica 28,6 ninfas fêmea ${ }^{-1}$. Valores mais elevados $\left(31,95\right.$ ninfas fêmea $\left.^{-1}\right)$ foram obtidos por Camargo (2007), utilizando colônias monoclonais de $C$. atlantica, a $20^{\circ} \mathrm{C}$, em mudas adubadas com teor médio de $30,0 \mathrm{~N} \mathrm{~kg}^{-1}$ de acículas de Pinus taeda. Os dois parâmetros são semelhantes aos observados no presente estudo, para as mudas dos viveiros comerciais das colônias multi e monoclonal. Ottati (2004) observou para C. atlantica a $22^{\circ} \mathrm{C}$ e Camargo (2007) a $20^{\circ} \mathrm{C}$, porém em mudas não adubadas, valores de 10,9 e 21,2 ninfas fêmea ${ }^{-1}$, respectivamente. Mustafa (1987) registrou para C. maritimae (Duffor, 1833 ) e para $C$. cupressi (Buckton, 1881 ), a $20^{\circ} \mathrm{C}$, valores de 13,8 e 23,5 ninfas fêmea ${ }^{-1}$, respectivamente. Todos estes valores assemelham-se a alguns tratamentos das progênies (T1, T3, T4, T5 e T6). Porém, os resultados diferem dos obtidos por Van Rensburg (1981), para $C$. cronartii (Tissot \& Pepper, 1967), de 67 ninfas fêmea ${ }^{-1}$.

Tabela 1. Fecundidade média (dias) \pm EP (erro padrão) de fêmeas originárias de colônias multiclonais de Cinara atlantica criadas em mudas de Pinus taeda de diferentes origens.

\begin{tabular}{|c|c|c|c|c|}
\hline Tratamentos & $\begin{array}{c}\mathrm{N}^{0} \text { ninfas fêmea } \\
\text { Média }^{-1} \mathrm{EP}\end{array}$ & $\begin{array}{l}\text { Redução em relação } \\
\text { ao maior valor (\%) }\end{array}$ & $\begin{array}{c}\mathrm{N}^{0} \text { ninfas fêmea } \\
\text { Média } \pm \mathrm{EP}\end{array}$ & $\begin{array}{l}\text { Redução em relação ao } \\
\text { maior valor }(\%)\end{array}$ \\
\hline $\mathrm{T} 1$ - viveiro RS & $29,9 \pm 6,5 b$ & - & $2,9 \pm 0,6 \mathrm{ab}$ & 23,7 \\
\hline $\mathrm{T} 2$ - viveiro $\mathrm{PR}$ & $25,4 \pm 4,3 b$ & 15,1 & $3,8 \pm 0,6 b$ & - \\
\hline T3 - progênie 1 & $10,8 \pm 3,5 \mathrm{a}$ & 63,9 & $1,0 \pm 0,3 \mathrm{a}$ & 73,7 \\
\hline T4 - progênie 2 & $24,5 \pm 4,2 \mathrm{ab}$ & 18,1 & $1,5 \pm 0,2 \mathrm{a}$ & 60,5 \\
\hline T5 - progênie 3 & $21,2 \pm 3,5 \mathrm{ab}$ & 29,1 & $1,3 \pm 0,2 \mathrm{a}$ & 65,8 \\
\hline T6 - progênie 4 & $16,7 \pm 3,4 \mathrm{ab}$ & 44,1 & $1,7 \pm 0,3 \mathrm{a}$ & 55,3 \\
\hline $\mathrm{T} 7$ - progênie 5 & $17,5 \pm 3,1 \mathrm{ab}$ & 41,5 & $1,3 \pm 0,2 \mathrm{a}$ & 65,8 \\
\hline
\end{tabular}

Médias seguidas pelas mesmas letras, nas colunas não diferem entre si pelo teste de Duncan ao nível de $5 \%$ de probabilidade. Número de insetos utilizados por tratamento: $\mathrm{T} 1-20 ; \mathrm{T} 2-25 ; \mathrm{T} 3-24 ; \mathrm{T} 4-18 ; \mathrm{T} 5-23 ; \mathrm{T} 6-22$ e $\mathrm{T} 7-24$. Temperatura de $20 \pm 2{ }^{\circ} \mathrm{C}$, umidade relativa de $70 \pm 10 \%$ e fotofase de $14 \mathrm{~h}$.

Tabela 2. Fecundidade média (dias) \pm EP (erro padrão) de fêmeas originárias de colônias monoclonais de Cinara atlantica e criadas em mudas de Pinus taeda de diferentes origens.

\begin{tabular}{|c|c|c|c|c|}
\hline Tratamentos & $\begin{array}{c}\mathrm{N}^{0} \text { ninfas fêmea } \\
\text { Média }^{-1} \mathrm{EP}\end{array}$ & $\begin{array}{l}\text { Redução em relação } \\
\text { ao maior valor (\%) }\end{array}$ & $\begin{array}{c}\mathrm{N}^{0} \text { ninfas fêmea } \\
\text { Média } \pm \text { EP }\end{array}$ & $\begin{array}{l}\text { Redução em relação ao } \\
\text { maior valor (\%) }\end{array}$ \\
\hline $\mathrm{T} 1$ - viveiro RS & $30,6 \pm 3,5 b$ & - & $1,9 \pm 0,2 \mathrm{c}$ & - \\
\hline T3 - progênie 1 & $11,3 \pm 3,4 \mathrm{a}$ & 63,1 & $1,6 \pm 0,5 b$ & 15,8 \\
\hline T4 - progênie 2 & $6,9 \pm 1,9 \mathrm{a}$ & 77,5 & $0,9 \pm 0,3 \mathrm{a}$ & 52,6 \\
\hline T5 - progênie 3 & $13,5 \pm 2,4 \mathrm{a}$ & 55,9 & $1,4 \pm 0,3 \mathrm{ab}$ & 26,3 \\
\hline T6 - progênie 4 & $10,7 \pm 2,9 \mathrm{a}$ & 65,0 & $1,1 \pm 0,3 \mathrm{ab}$ & 42,1 \\
\hline $\mathrm{T} 7$ - progênie 5 & $7,0 \pm 0,9 \mathrm{a}$ & 77,1 & $1,1 \pm 0,1 \mathrm{ab}$ & 42,1 \\
\hline
\end{tabular}

Médias seguidas pelas mesmas letras, nas colunas não diferem entre si pelo teste de Duncan ao nível de 5\% de probabilidade; Número de insetos utilizados por tratamento: $\mathrm{T} 1-19 ; \mathrm{T} 3-21 ; \mathrm{T} 4-19 ; \mathrm{T} 5-32 ; \mathrm{T} 6-23$ e T7 -23 . Temperatura de $20 \pm 2^{\circ} \mathrm{C}$, umidade relativa de $70 \pm 10 \%$ e fotofase de $14 \mathrm{~h}$.

A produção diária de ninfas fêmea-1, para os insetos multiclonais, foi maior nas mudas dos viveiros (T1) e (T2), porém o T2 foi o que diferiu das progênies, com produção muito superior (Tabela 1). Os insetos monoclonais apresentaram a maior produção de ninfas fêmea ${ }^{-1} \operatorname{dia}^{-1}$ no $\mathrm{T} 1(1,9)$, diferindo estatisticamente dos demais tratamentos (Tabela 2). Este valor é semelhante ao observado por Khaemba \& Wanjala (1993) para C. cupressi, que produziu 2 ninfas fêmea-1 dia $^{-1}$. Camargo (2007), 
utilizando colônias monoclonais de $C$. atlantica, a $20^{\circ} \mathrm{C}$, porém em mudas adubadas com teor médio de $30,0 \mathrm{~N} \mathrm{~kg}^{-1}$ de acículas de $P$. taeda, observou uma produção de 1,62 ninfas fêmea ${ }^{-1}$ dia $^{-1}$. Entretanto, no tratamento controle (sem adubação), o valor obtido (1,14 ninfas fêmea ${ }^{-1}$ dia $\left.^{-1}\right)$ assemelha-se aos obtidos nas progênies deste estudo (Tabelas $1 \mathrm{e}$ 2). O efeito positivo de diferentes concentrações de nitrogênio na reprodução também foi relatado por Hogendorp et al. (2006) em Planococcus citri (cochonilha de citrus) e em C. atlantica por Camargo (2007). Hogendorp et al. (2006) verificaram que altas concentrações de nitrogênio nas plantas de Solenostemon scutellarioides são os responsáveis pela alta capacidade reprodutiva, pelo grande tamanho das fêmeas e pelo curto tempo de desenvolvimento da cochonilha de citrus. Camargo (2007) observou que adultos de $C$. atlantica mantidos em plantas de P. taeda com maior concentração de nitrogênio, apresentaram maior tamanho e maior produção de ninfas.

Verificou-se que as mudas dos viveiros comerciais favoreceram a reprodução de $C$. atlantica. Estudos com outras espécies corroboram com a observação de que progênies ou cultivares de plantas podem apresentar diferenças na resistência ao ataque de afídeos. Bertin et al. (2013), estudando o efeito da planta hospedeira sobre o desenvolvimento, sobrevivência e reprodução de Dysmicoccus brevipes (Hemiptera: Pseudococcidae), concluíram que o hospedeiro pode afetar o desenvolvimento e sobrevivência do inseto e que o uso apropriado de uma cultivar resistente pode ajudar na redução da população da praga, auxiliando no manejo integrado da praga. Schuster \& Starks (1973) verificaram que Schizaphis graminum (Rondani, 1852), criado em cultivar resistente de sorgo, apresentou redução de 77,7\% no número de ninfas fêmea ${ }^{-1}$.

Para os insetos de colônias multiclonais, mantidas nas mudas das progênies, verificou-se que a redução no número de ninfas fêmea-1 ${ }^{-1}$ em relação ao T1 (maior valor), variou de $18,1 \%$ (T4) a 63,9\% (T3) (Tabela 1). Para os insetos de colônias monoclonais, a redução foi menos variável (55,9\% a 77,5\%) (Tabela 2).

Kamunya et al. (2008) observaram em 18 diferentes famílias de Cupressus lusitanica Mill., que a resistência ao ataque de Cinara cupressi (Buckton, 1881) apresentou variação significativa entre e dentro das famílias, mas que houve um forte controle genético, fator importante para o desenvolvimento de programas de melhoramento genético. Vîlcan et al. (2013) estudaram a resistência de diferentes clones de Larix sp. ao ataque de Adelges laricis Vall. (Homoptera, Adelgidae) e observaram que a resistência ou susceptibilidade dos clones ao ataque do inseto foi fortemente influenciada pelo genótipo. Uma vez que as progênies são fruto de uma seleção genética, é possível haver algum fator de resistência interferindo no desenvolvimento de $C$. atlantica nestas plantas.

Comparando-se os insetos das colônias multi e monoclonais (Tabelas 1 e 2), deduz-se que a primeira favoreceu o desenvolvimento do afídeo, resultando em um maior número de descendentes, principalmente nas progênies, fator importante quando o objetivo é a manutenção da criação do inseto em laboratório para estudos biológicos.

Os insetos das colônias multiclonais apresentaram os maiores valores para os parâmetros morfométricos, para todos os tratamentos, com exceção do comprimento do tarsômero II, que foi muito semelhante nos dois tipos de criação (Tabela 3 ).

Nas colônias monoclonais, em todos os tratamentos e para a maioria dos parâmetros, os valores foram menores aos observados por Camargo (2007), embora o autor também tenha utilizado insetos de criação monoclonal. Para os multiclonais, foi observada uma grande variação nos valores dos parâmetros analisados, sendo que alguns são maiores, outros menores e outros muito semelhantes aos observados por Camargo (2007). Kairo \& Murphy (1999) obtiveram para $C$. cupressivora, em Cupressus macrocarpa, valores de $1,82 \mathrm{~mm}$ e $0,89 \mathrm{~mm}$, para os comprimentos do corpo e da tíbia posterior, respectivamente. Para o comprimento do corpo, verifica-se semelhança com os resultados obtidos para C. atlantica, apenas nos tratamentos $3 \mathrm{e}$ 7 da criação monoclonal, sendo que os demais foram todos superiores. Com relação ao comprimento da tíbia posterior, apenas o $\mathrm{T} 1$ apresentou valor próximo ao de Camargo (2007) para C. atlantica. Para os demais tratamentos os valores foram inferiores. Entretanto, os valores foram superiores aos obtidos por Kairo \& Murphy (1999) para C. cupressivora (Tabela 3). 
Tabela 3. Dados morfométricos de fêmeas partenogenéticas adultas procedentes de colônias multi e monoclonais de Cinara atlantica mantidas em mudas de Pinus taeda de diferentes origens.

\begin{tabular}{|c|c|c|c|c|c|c|c|c|}
\hline $\begin{array}{c}\text { Caracteres } \\
(\mathrm{mm})\end{array}$ & $\begin{array}{l}\text { Tipo de } \\
\text { criação }\end{array}$ & $\begin{array}{c}\text { T1 } \\
\text { Média } \pm \text { EP }\end{array}$ & $\begin{array}{c}\text { T2 } \\
\text { Média } \pm \text { EP }\end{array}$ & $\begin{array}{c}\text { T3 } \\
\text { Média } \pm \mathbf{E P}\end{array}$ & $\begin{array}{c}\text { T4 } \\
\text { Média } \pm \text { EP }\end{array}$ & $\begin{array}{c}\text { T5 } \\
\text { Média } \pm \text { EP }\end{array}$ & $\begin{array}{c}\text { T6 } \\
\text { Média } \pm \text { EP }\end{array}$ & $\begin{array}{c}\text { T7 } \\
\text { Média } \pm \text { EP }\end{array}$ \\
\hline \multirow{2}{*}{$\begin{array}{l}\text { Comprimento } \\
\text { corpo }\end{array}$} & Multiclonal & $2,69 \pm 0,17$ & $2,47 \pm 0,12$ & $2,02 \pm 0,15$ & $2,50 \pm 0,19$ & $2,08 \pm 0,15$ & $2,31 \pm 0,05$ & $2,39 \pm 0,10$ \\
\hline & Monoclonal & $2,31 \pm 0,11$ & - & $1,81 \pm 0,14$ & $1,97 \pm 0,22$ & $1,98 \pm 0,08$ & $1,92 \pm 0,17$ & $1,76 \pm 0,06$ \\
\hline \multirow{2}{*}{ Largura cabeça } & Multiclonal & $0,65 \pm 0,01$ & $0,65 \pm 0,03$ & $0,57 \pm 0,02$ & $0,64 \pm 0,01$ & $0,58 \pm 0,03$ & $0,62 \pm 0,00$ & $0,61 \pm 0,01$ \\
\hline & Monoclonal & $0,59 \pm 0,01$ & - & $0,55 \pm 0,02$ & $0,55 \pm 0,02$ & $0,57 \pm 0,01$ & $0,51 \pm 0,03$ & $0,51 \pm 0,01$ \\
\hline \multirow{2}{*}{$\begin{array}{l}\text { Comprimento } \\
\text { antena }\end{array}$} & Multiclonal & $1,21 \pm 0,04$ & $1,16 \pm 0,06$ & $1,00 \pm 0,05$ & $1,19 \pm 0,06$ & $1,03 \pm 0,08$ & $1,10 \pm 0,02$ & $1,08 \pm 0,06$ \\
\hline & Monoclonal & $1,08 \pm 0,03$ & - & $0,89 \pm 0,05$ & $0,97 \pm 0,04$ & $0,98 \pm 0,03$ & $0,88 \pm 0,06$ & $0,84 \pm 0,02$ \\
\hline \multirow{2}{*}{$\begin{array}{l}\text { Comprimento } \\
\text { artículo rostral } \\
\mathrm{IV}+\mathrm{V}\end{array}$} & Multiclonal & $0,27 \pm 0,00$ & $0,25 \pm 0,01$ & $0,34 \pm 0,04$ & $0,35 \pm 0,06$ & $0,25 \pm 0,01$ & $0,30 \pm 0,03$ & $0,23 \pm 0,01$ \\
\hline & Monoclonal & $0,25 \pm 0,01$ & - & $0,23 \pm 0,00$ & $0,24 \pm 0,01$ & $0,24 \pm 0,01$ & $0,23 \pm 0,01$ & $0,22 \pm 0,01$ \\
\hline \multirow{2}{*}{$\begin{array}{l}\text { Comprimento } \\
\text { fêmur posterior }\end{array}$} & Multiclonal & $1,09 \pm 0,05$ & $1,09 \pm 0,05$ & $0,81 \pm 0,07$ & $1,05 \pm 0,07$ & $0,87 \pm 0,09$ & $0,96 \pm 0,03$ & $0,89 \pm 0,05$ \\
\hline & Monoclonal & $0,94 \pm 0,04$ & - & $0,73 \pm 0,08$ & $0,82 \pm 0,05$ & $0,83 \pm 0,05$ & $0,74 \pm 0,06$ & $0,63 \pm 0,03$ \\
\hline \multirow{2}{*}{$\begin{array}{l}\text { Comprimento } \\
\text { tíbia posterior }\end{array}$} & Multiclonal & $1,91 \pm 0,10$ & $1,97 \pm 0,08$ & $1,43 \pm 0,10$ & $1,83 \pm 0,11$ & $1,56 \pm 0,15$ & $1,65 \pm 0,04$ & $1,51 \pm 0,11$ \\
\hline & Monoclonal & $1,62 \pm 0,07$ & - & $1,27 \pm 0,12$ & $1,41 \pm 0,09$ & $1,45 \pm 0,08$ & $1,29 \pm 0,10$ & $1,15 \pm 0,04$ \\
\hline \multirow{2}{*}{$\begin{array}{l}\text { Comprimento } \\
\text { tarsômero II }\end{array}$} & Multiclonal & $0,22 \pm 0,01$ & $0,22 \pm 0,01$ & $0,19 \pm 0,00$ & $0,25 \pm 0,01$ & $0,20 \pm 0,01$ & $0,22 \pm 0,01$ & $0,21 \pm 0,01$ \\
\hline & Monoclonal & $0,22 \pm 0,00$ & - & $0,21 \pm 0,01$ & $0,24 \pm 0,02$ & $0,21 \pm 0,01$ & $0,20 \pm 0,01$ & $0,21 \pm 0,01$ \\
\hline
\end{tabular}

T1 e T2- mudas de viveiro do RS e PR, respectivamente; T3, T4, T5, T6 e T7 - progênies de $P$. taeda cedidas por empresa florestal de SC. EP = erro padrão.

Para estudar a relação existente entre os parâmetros, foram calculados os coeficientes de correlação (Tabela 4). Verificou-se que os coeficientes de correlação abaixo de 0,33 não foram significativos pelo teste $t$, ao nível de $0,1 \%$ de probabilidade. Esses valores ocorreram entre o comprimento dos artículos rostrais IV $+\mathrm{V}$ com os comprimentos do corpo, do fêmur, da tíbia e do tarsômero II. Porém, no processo de seleção final, visando à obtenção de uma variável representativa do tamanho do corpo do inseto, adotou-se como critério o rigor de considerar apenas os coeficientes de correlação superiores a 0,80 . Assim, foram excluídas, nesta etapa da análise de componentes principais, as variáveis comprimento dos artículos rostrais $\mathrm{IV}+\mathrm{V}$ e comprimento do tarsômero II.

A análise dos componentes principais (Tabela 5) indicou que os três primeiros vetores explicam $98 \%$ da variação total. Assim, as mensurações do comprimento da antena, da tíbia e do fêmur posterior correspondem aos maiores valores absolutos dos coeficientes de cada um dos dois primeiros vetores, dispensando a utilização das demais variáveis inicialmente avaliadas.

Tabela 4. Coeficientes de correlação entre os parâmetros morfométricos de fêmeas partenogenéticas adultas originárias de colônias multi e monoclonais de Cinara atlantica mantidas nas mudas de viveiros comerciais e nas progênies de Pinus taeda.

\begin{tabular}{|c|c|c|c|c|c|c|}
\hline Caracteres & $\begin{array}{l}\text { Larg. } \\
\text { cabeça }\end{array}$ & $\begin{array}{l}\text { Comp. } \\
\text { antena }\end{array}$ & $\begin{array}{l}\text { Comp. art. } \\
\text { rostral V+V }\end{array}$ & Comp. fêmur & Comp. tibia & $\begin{array}{c}\text { Comp. } \\
\text { tarsômero II }\end{array}$ \\
\hline Comprimento do corpo & 0,84 & 0,84 & 0,28 & 0,83 & 0,83 & 0,44 \\
\hline Largura da cabeça & & 0,91 & 0,37 & 0,88 & 0,87 & 0,57 \\
\hline $\begin{array}{l}\text { Comprimento da } \\
\text { antena }\end{array}$ & & & 0,33 & 0,93 & 0,91 & 0,53 \\
\hline $\begin{array}{l}\text { Comp.artículos rostral } \\
\text { IV }+ \text { V }\end{array}$ & & & & 0,28 & 0,26 & 0,22 \\
\hline Comp. fêmur posterior & & & & & 0,98 & 0,54 \\
\hline Comp. tíbia posterior & & & & & & 0,53 \\
\hline
\end{tabular}


Tabela 5. Componentes principais dos parâmetros de maior importância na determinação do tamanho de fêmeas partenogenéticas adultas de colônias multi e monoclonais de Cinara atlantica mantidas nas mudas de viveiros comerciais e nas progênies de Pinus taeda.

\begin{tabular}{cccc}
\hline Vetor & Auto-valores & Variância relativa & $\begin{array}{c}\text { Variância } \\
\text { acumulada }\end{array}$ \\
\hline 1 & 4,5286 & 90,57 & 90,57 \\
2 & 0,2220 & 4,44 & 95,01 \\
3 & 0,1551 & 3,10 & 98,11 \\
4 & 0,0770 & 1,54 & 99,66 \\
5 & 0,0171 & 0,34 & 100,00 \\
\hline
\end{tabular}

Para a definição das três variáveis mais importantes, foram analisados os valores dos auto-vetores (Tabela 6) e partiu-se para a eliminação das variáveis de menor importância. Assim, na sequência do processo, eliminou-se inicialmente a variável comprimento do corpo, que apresentou o maior valor; posteriormente foi eliminada a variável largura da cabeça; ficando como terceiro componente a variável comprimento do fêmur posterior; como segundo, a variável comprimento da tíbia posterior; e como componente número um, a variável comprimento da antena (Tabela 6).

Tabela 6. Auto-vetores dos componentes principais dos parâmetros morfológicos de insetos adultos de colônias multi e monoclonais de Cinara atlantica mantidos nas mudas de viveiros comerciais e nas progênies de Pinus taeda.

\begin{tabular}{lccccc}
\hline \multicolumn{1}{c}{ Variável } & Vetor & Vetor & Vetor & Vetor & Vetor \\
& $\mathbf{1}$ & $\mathbf{2}$ & $\mathbf{3}$ & $\mathbf{4}$ & $\mathbf{5}$ \\
\hline Comprimento do corpo & 0,828492 & 0,976986 & 0,999996 & 1,000000 & 1,000000 \\
Largura da cabeça & 0,893107 & 0,896360 & 0,981659 & 0,999992 & 1,000000 \\
Comprimento da antena & 0,9313640 & 0,933871 & 0,947149 & 0,999740 & 1,000000 \\
Comp. fêmur posterior & 0,945453 & 0,976835 & 0,989862 & 0,990420 & 1,000000 \\
Comp. tíbia posterior & 0,930150 & 0,966584 & 0,987106 & 0,992701 & 1,000000 \\
\hline
\end{tabular}

Com os valores das três principais variáveis foi criada uma nova variável denominada "tamanho do inseto", que foi utilizada para comparação entre tratamentos.

Camargo (2007), avaliando a morfometria de adultos de $C$. atlantica em plantas de $P$. taeda adubadas com diferentes concentrações de nitrogênio, verificou que os parâmetros que apresentaram valores significativos foram o comprimento do corpo, antena, fêmur, tíbia e diâmetro do sifúnculo. Kairo \& Murphy (1999), quando avaliaram o efeito da temperatura no tamanho de $C$. cupressivora, verificaram uma relação negativa entre o comprimento do corpo e o aumento da temperatura e que, para o comprimento da tíbia posterior, não ocorreu diferença significativa. No presente trabalho, o comprimento do corpo foi uma variável que apresentou baixa importância, ao contrário do comprimento da tíbia posterior, que foi uma das variáveis mais representativas do tamanho do inseto. Carter et al. (1991) e Awmack \& Leather (2002) relataram que o comprimento da tíbia posterior está fortemente correlacionado com a fecundidade do inseto.

Na Tabela 7 é apresentada a comparação da nova variável, "tamanho do inseto", entre os tratamentos. Para os insetos provenientes de colônias multiclonais, verificou-se que nas mudas dos viveiros (T1 e T2), os insetos apresentaram o maior tamanho $(3,99 \mathrm{~mm}$ e 4,0 $\mathrm{mm}$ ) e foram estatisticamente iguais, não diferindo dos tratamentos 4 e 6, embora o nível de significância do T6 com estes tratamentos tenha sido de apenas 0,18 e 0,19, respectivamente, indicando uma menor semelhança entre eles. Entre as progênies, onde foram observados os menores insetos, o T3 diferiu do T4 e este foi diferente do T5 e T7 porém com um nível de significância de apenas 0,12 . Os insetos monoclonais apresentaram menor tamanho que os multiclonais, tendo o $\mathrm{T} 1$ a maior média $(3,53 \mathrm{~mm})$ e igual aos insetos das progênies 4 e 5 , porém, também com baixos níveis de significância $(0,15$ e 0,20 , respectivamente). Entre as progênies, apenas as 4 e 5 foram diferentes da 7 (Tabela 7). 
Tabela 7. Valores médios da variável "tamanho do inseto" e níveis de significância entre tratamentos, pelo teste de Duncan, obtidos para Cinara atlantica mantidas em mudas de viveiros comerciais e de progênies de Pinus taeda.

\begin{tabular}{|c|c|c|c|c|c|c|c|}
\hline \multicolumn{8}{|c|}{ Colônias multiclonais } \\
\hline & T1 & $\mathbf{T 2}$ & T3 & T4 & T5 & T6 & $\mathbf{T} 7$ \\
\hline Média & 3,99 & 4,0 & 3,07 & 3,85 & 3,28 & 3,51 & 3,29 \\
\hline $\mathrm{T} 1$ & & 0,98 & 0,02 & 0,68 & 0,06 & 0,18 & 0,06 \\
\hline $\mathrm{T} 2$ & & & 0,02 & 0,69 & 0,06 & 0,19 & 0,06 \\
\hline $\mathrm{T} 3$ & & & & 0,04 & 0,54 & 0,24 & 0,55 \\
\hline $\mathrm{T} 4$ & & & & & 0,12 & 0,31 & 0,12 \\
\hline T5 & & & & & & 0,52 & 0,97 \\
\hline T6 & & & & & & & 0,51 \\
\hline \multicolumn{8}{|c|}{ Colônias monoclonais } \\
\hline Média & 3,53 & - & 2,80 & 3,10 & 3,16 & 2,82 & 2,54 \\
\hline $\mathrm{T} 1$ & & - & 0,03 & 0,15 & 0,20 & 0,03 & 0,003 \\
\hline $\mathrm{T} 3$ & & - & & 0,34 & 0,26 & 0,93 & 0,37 \\
\hline $\mathrm{T} 4$ & & - & & & 0,82 & 0,35 & 0,08 \\
\hline T5 & & - & & & & 0,27 & 0,06 \\
\hline $\mathrm{T} 6$ & & - & & & & & 0,36 \\
\hline
\end{tabular}

T1 e T2- mudas de viveiro do RS e PR, respectivamente; T3, T4, T5, T6 e $\mathrm{T} 7$ - progênies de $P$. taeda cedidas por empresa florestal de SC.

Nas Figuras 2 e 3 são apresentados os valores obtidos para a variável "tamanho do inseto" e fecundidade nos diferentes tratamentos, comparando os insetos provenientes de colônias multi e monoclonais. Observou-se que nos insetos da criação multiclonal ocorreram os maiores valores para as duas variáveis avaliadas, quando comparado com os insetos da criação monoclonal, com exceção do tratamento T1 da criação monoclonal que foi praticamente igual ao T1 e T2 da criação multiclonal, sendo as mudas destes tratamentos todas originárias de viveiros comerciais.

Verificou-se que, para os dois tipos de criação, as mudas dos viveiros comerciais apresentaram os maiores insetos e maior produção de ninfas, quando comparado às progênies.

Kennedy \& Kishaba (1977) observaram que a redução da fecundidade total e diária e do tamanho dos afídeos pode ser uma consequência da não preferência do inseto por um determinado hospedeiro, fato este observado para os adultos de $C$. atlantica mantidos nas mudas das progênies, indicando uma não preferência destes afídeos por este tipo de muda.

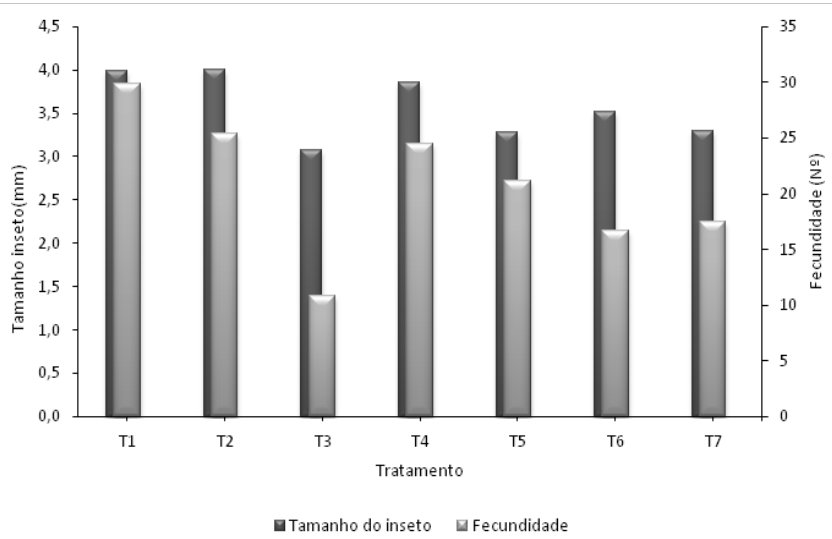

Figura 2. Valores da fecundidade e "tamanho do inseto" obtidos para adultos de Cinara atlantica em mudas de viveiros comerciais e de progênies de Pinus taeda, em colônias multiclonais. T1 e T2- mudas de viveiro do RS e PR, respectivamente; T3, T4, T5, T6 e T7 - progênies de $P$. taeda cedidas por empresa florestal de SC.

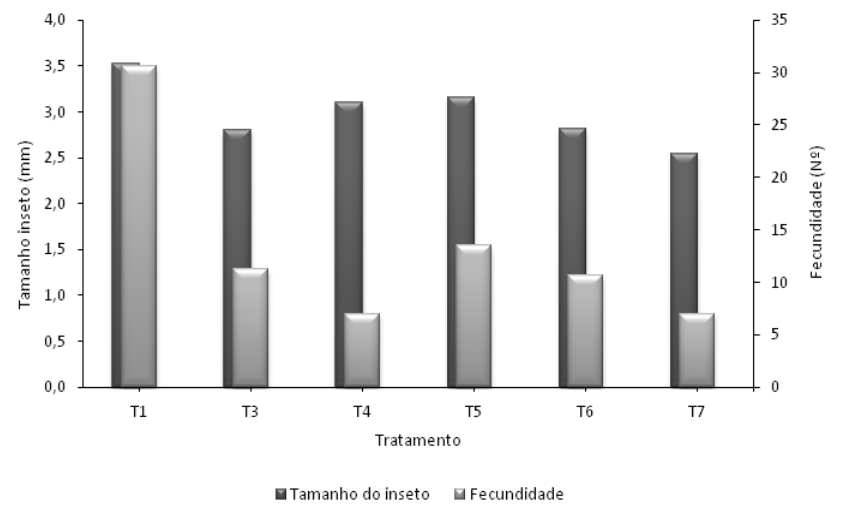

Figura 3. Valores da fecundidade e "tamanho do inseto" obtidos para adultos de Cinara atlantica em mudas de viveiros comerciais e de progênies de Pinus taeda, em colônias monoclonais. T1 e T2- mudas de viveiro do RS e PR, respectivamente; T3, T4, T5, T6 e T7 - progênies de $P$. taeda cedidas por empresa florestal de SC.

\section{Conclusões}

As mudas de viveiros comerciais apresentam-se mais favoráveis para o desenvolvimento de Cinara atlantica que as mudas das progênies de Pinus taeda testadas neste trabalho, resultando em insetos de maior tamanho e com mais descendentes. 
O comprimento da tíbia posterior, fêmur posterior e antena são os parâmetros morfométricos que apresentam maior correlação com o tamanho dos insetos, sendo as variáveis mais importantes e que representam $98 \%$ da variação total.

Os insetos provenientes de colônias multiclonais apresentaram, para a maioria dos parâmetros morfométricos avaliados, os maiores valores e também a maior produção de descendentes, provavelmente em razão da maior variabilidade genética em função das ninfas serem originadas de diferentes fềmeas. Sendo assim, a utilização de insetos procedentes de colônias multiclonais é mais adequada para a manutenção de criações em laboratório, quando comparada com as monoclonais.

A menor suscetibilidade das mudas das progênies pode ser comprovada pela redução da fertilidade total e diária, redução do tamanho dos insetos e maior mortalidade de C. atlantica, o que as torna mais adequadas do que as mudas de viveiros comerciais para programas de manejo que incluam linhagens resistentes.

\section{Referências}

Awmack, C. S \& Leather, S. R. Host plant quality and fecundity in herbivorous insects. Annual Review of Entomology, v. 47, p. 817-844, 2002. DOI: 10.1146/annurev.ento.47.091201.145300.

Begon, M. et al. Ecology: individuals, populations and communities. Oxford: Blackwell Scientific Publications, 1986, 876 p.

Bertin, A. et al. Host plant effects on the development, survival, and reproduction of Dysmicoccus brevipes (Hemiptera: Pseudococcidae) on Grapevines. Annals of the Entomological Society of America, v. 106, n. 5, p. 604-609, 2013. DOI: 10.1603/AN13030.

Camargo, J. M. M. C. Efeito da aplicação de nitrogênio e silício em plantas de Pinus taeda L. (Pinaceae) na performance do pulgãogigante-do-pinus, Cinara atlantica (Wilson, 1919) (Hemíptera: Aphididae). 2007. 101 f. Dissertação (Mestrado em Ciências Biológicas) - Universidade Federal do Paraná, Curitiba.

Carter, C. \& Watson, G. The ecology of conifer aphids and its bearing on forest establishment and productivity. In: WORKSHOP PROCEEDINGS: exotic aphid pests of conifers: a crisis in African forestry. Rome: FAO, 1991. 160 p. Available from: <http://www.fao. org/docrep/008/u4778e/U4778E00.htm>. Access on: 10 set 2013.

Carter, M. R. et al. Changes in gypsy moth (Lepidoptera, Lymantriidae) fecundity and male wing length resulting from defoliation. Environmental Entomology, n. 20, p. 1042-1047, 1991. DOI: $10.1093 / \mathrm{ee} / 20.4 .1042$.

Carver, M. et al. Hemiptera. In: CSIRO (Australia). Division of Entomology. The insects of Australia. [Canberra], 1991. p. 429-509.
Dixon, A. F. G. Aphid ecology an optimization approach. 2nd ed. London: Chapman and Hall, 1998. 300 p.

Dixon, A. F. G. Parthenogenetic reproduction and the rate of increase in aphids. In: Minks, A. K. \& Harrewinjn, P. World crop pests: Aphids: their biology, natural enemies and control. Amsterdam: Elsevier, 1987. p. 269-287.

Hogendorp, B. K. et al. Effect of nitrogen fertility on reproduction and development of citrus mealybug, Planococcus citri Risso (Homoptera: Pseudococcidae), feeding on two colors of coleus, Solenostemon scutellarioides L. Codd. Environmental Entomology, n. 35, p. 201-211, 2006. DOI: 10.1603/0046-225X-35.2.201.

Kairo, M. T. K. \& Murphy, S. T. Temperature and plant nutrient effects on the development, survival and reproduction of Cinara sp. nov., an invasive pest of cypress trees in Africa. Entomologia Experimentalis et Applicata, n. 92, p. 147-156, 1999. DOI: 10.1046/j.1570-7458.1999.00534.x.

Kamunya, S. M. et al. Variation and inheritance of resistance to cypress aphid, Cinara cupressi Buckton in Cupressus lusitanica Miller. Annals of Applied Biology, v. 130, n. 1, p. 27-36, 2008. DOI:10.1111/j.1744-7348.1997.tb05780.x.

Kennedy, G. G. \& Kishaba, A. N. Response of alate melon aphids to resistant and susceptible muskmelon lines. Journal of Economic Entomology, n. 70, p. 407-410, 1977. DOI: 10.1093/jee/70.4.407.

Khaemba, B. M. \& Wanjala, F. M. E. Some aspects of the biology of Cinara cupressi Buckton (Homoptera: Lachnidae) when bred under laboratory condition. Insect Science and its Application, n. 14, p. 693-695, 1993. DOI: 10.1017/S1742758400018130.

Kocourek, F. et al. Effect of temperature on development rate and intrinsic rate of increase of Aphis gossypii reared on greenhouse cucumbers. Entomologia Experimentalis et Applicata, n. 71, p. 59-64, 1994. DOI: 10.1111/j.1570-7458.1994.tb01769.x.

Mustafa, T. M. Reproductive biology and population studies of cypress aphid Cinara cupressi (Buckton) and pine aphid Cinara maritimae (Dafour). Dirasat Online, n. 14, p. 99-105, 1987.

Ottati, A. L. T. Aspectos bioecológicos do pulgão-gigante-do-pinus, Cinara atlantica Wilson, 1919) (Hemiptera: Aphididae), em Pinus spp. (Pinaceae). 2004. 133 f. Tese (Doutorado em Agronomia) Universidade Estadual Paulista "Julio de Mesquita Filho", Botucatu.

Penteado, S. R. C. et al. Pulgão do Pinus: nova praga florestal. Série Técnica do IPEF. v. 13, n. 33, p. 97-102, 2000.

Schuster, D. J. \& Starks, K. J. Greenbugs: components of host-plant resistance in sorghum. Journal of Economic Entomology, n. 66, p. 1131-1134, 1973.

Traicevski, V. \& Ward, S. A. Birth weight and the rate of increase in the cowpea aphid Aphis craccivora. European Journal of Entomology, n. 91, p. 37-46, 1994.

Van Rensburg, N. J. A technique for rearing the black pine aphid, Cinara cronartii T\& P, and some features of its biology (Homoptera: Aphididade). Journal of Entomological Society of Southern Africa, v. 44, n. 2, p. 367-79, 1981.

Vîlcan, A. et al. The variability of different larch clone provenances on the response to the attack by its main pests and fungal diseases. Trees, v. 27, p. 697-705, 2013. DOI: 10.1007/s00468-012-0825-1. 
Zaleski, S. R. M. Biologia, danos e determinação dos limites térmicos para o desenvolvimento de Cinara atlantica (Wilson, 1919) (Hemiptera: Aphididae) em Pinus taeda L. (Pinaceae).

2003.70 f. Dissertação (Mestrado em Ciências Biológicas) Universidade Federal do Paraná, Curitiba. 